\title{
Cascade overlap with vacancy-type defects in Fe $\mathbf{e}^{\star, \star \star}$
}

\author{
Fredric Granberg ${ }^{\mathrm{a}}$, Jesper Byggmästar, and Kai Nordlund \\ Department of Physics, University of Helsinki, P.O. Box 43, FIN-00014 Helsinki, Finland
}

Received 29 April 2019 / Received in final form 14 May 2019

Published online 3 July 2019

(C) The Author(s) 2019. This article is published with open access at Springerlink.com

\begin{abstract}
In order to understand the effect of irradiation on the material properties, we need to look into the atomistic evolution of the system during the recoil event. The nanoscale features formed due to irradiation will ultimately affect the macroscopic properties of the material. The defect production in pristine materials have been subject to investigation previously, but as the dose increases, overlap will start to happen. This effect of cascades overlapping with pre-existing debris has only recently been touched, and mainly been investigated for interstitial-type defects. We focus on vacancy-type defect clusters in BCC Fe and start a recoil event in their near vicinity. The final defect number as well as the transformation of the defect clusters are investigated, and their behaviour is related to the distance between the defect and the cascade centre. We found that for vacancy-type defects, the suppression of defect production is not as strong as previously observed for interstitial-type defects. The cascade-induced transformation, such as change in Burgers vector or creation of dislocations, was determined for all initial defect structures.
\end{abstract}

\section{Introduction}

In modern society, iron and iron alloys are the most widely used metals in structural applications. These materials are also used in nuclear power plants, where the harsh environment will over time degrade the properties of the material. The macroscopic property changes are usually based on the change and evolution of defects, starting at the atomistic scale. These changes can be small defect clusters forming and hindering the movement of dislocations, which make the material harder but also more brittle, or the defect structure can change from an immobile to a mobile state or vice versa. Consequently, a large number of experimental and computational studies have been carried out to study the defects introduced by irradiation $[1,2]$. Experimental measurements can reveal what kinds of defects are formed after exposure to irradiation [3,4]. On the other hand, most experimental techniques are limited to larger defect clusters and structures, as the resolution is usually limited to a few nanometers. In addition, the exact structure of the defects can be difficult to determine in the setups. To remedy this, atomistic simulations on the primary damage formation in iron have been carried out in the last few decades, see for instance $[1,2]$, and references

\footnotetext{
* Contribution to the Topical Issue "Multiscale Materials Modeling", edited by Yoji Shibutani, Shigenobu Ogata, and Tomotsugu Shimokawa.

${ }^{\star}$ Supplementary material in the form of one pdf file available from the Journal web page at https://doi.org/10.1140/epjb/e2019-100240-3

a e-mail: fredric.granberg@helsinki.fi
}

therein. As the name indicates, these simulations will follow the trajectories of individual atoms, and therefore the detailed structure of the produced defects can be studied.

Experimental studies have shown that as the dose and defect concentration increases during continuous irradiation, at some point the damage seems to saturate [5]. The explanation to the saturation is that the cascade will heal some of the previous damage, and therefore not as many new defects are produced. The irradiation can also, on the other hand, reorient or restructure the defect clusters. Prolonged irradiation of iron results in several types of defect clusters, including dislocation loops, both interstitial- and vacancy-type, as well as voids and interstitial-rich C15 Laves-phase clusters [3,4,6-8], the last one theoretically shown to be stable. All of these features will affect the dislocation movement in their own manner [9-15], and hence affect the material properties. The experimentally observed defect saturation has also been reproduced by computational means previously for metals [16-18]. In addition, several studies have focused on the effect of cascades hitting previous debris. Some studies were conducted on the overlap of two or three cascades $[19,20]$, and some studies started from perfect defect clusters, and investigated the effect of a cascade overlapping with them $[21,22]$. The latter ones focused on interstitial-type defect clusters only. For interstitial-type defects, the defect production was previously shown to follow a simple analytical function as a function of the degree of cascade overlap [21].

In this study, we investigate three different types of vacancy-rich defect clusters and their response to a recoil in their vicinity. Both the produced defects as well as the 
transformation of the initial defect clusters are investigated. Several different primary knock-on atom (PKA) energies, temperatures and defect sizes are studied to get a broader picture. These newly obtained results, in addition to previous ones on interstitial-type defects, can be used in larger-scale simulations to introduce more realistically the effects of cascade overlap.

\section{Methods}

The simulations were carried out with the classical MD code PARCAS [23,24], with an adaptive timestep [25]. To describe the interaction between the atoms, we used two different interatomic potentials, the Ackland et al. potential (A04) [26] and the Byggmästar et al. modification of the Marinica et al. potential (M07B) [18], based on the potential in $[8,27]$. These same potentials were used previously for the interstitial-type defect study [21], which enables using a combination of the results in future studies. Electronic stopping was applied on all atoms with a kinetic energy over $1 \mathrm{eV}$ [28], and was implemented as a friction force on the atoms. The simulation cell was

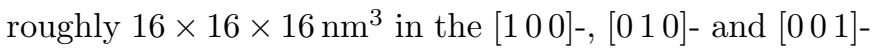
directions, for the void and $\langle 100\rangle$ loop case, and in the [1 $1 \overline{1} 0]-,[\overline{1} 12]-$ and [1 11 ]-directions, for the $1 / 2\langle 111\rangle$ loop case. The box contained about 330000 atoms. The recoils were initiated at different distances from the defect, and the direction was always towards the centre of the defect cluster. The different distances varied between $2 \mathrm{~nm}$ and $6 \mathrm{~nm}$, to get both full overlap as well as partial and zero overlap. The total simulation time was $30 \mathrm{ps}$, long enough for the system to cool down to its initial temperature, as a few layers of atoms at the borders were thermally controlled by a Berendsen thermostat [29].

Both $0 \mathrm{~K}$ and room temperature $(300 \mathrm{~K})$ were investigated for both interatomic potentials. Two PKA energies were studied, $5 \mathrm{keV}$ and $10 \mathrm{keV}$, to reveal any dependence on recoil energy. The different defect structures investigated were spherical voids, and vacancy-type $1 / 2\langle 111\rangle$ and $\langle 100\rangle$ loops. The loops were of three sizes, roughly 30,60 and 110 vacancies, corresponding to roughly $1.2 \mathrm{~nm}$, $1.8 \mathrm{~nm}$ and $2.5 \mathrm{~nm}$ in diameter $\left(d_{\text {def }}\right)$. The spherical voids were simulated in a total of 5 sizes (roughly 30, 60, 110, 260 and 610 vacancies), corresponding to the same diameters as the loops as well as to the same number of vacancies (diameters $0.7 \mathrm{~nm}$ and $0.9 \mathrm{~nm}$ ). In addition, single vacancies were also studied. For each energy, interatomic potential, temperature, defect type, and defect size, a total of 500 recoils were initiated (100 recoils per distance). The results on defect production and cascade volume in pristine materials for the considered temperatures, energies, and interatomic potentials were the average of 50 or 100 recoil events. Some of these are available in the literature (average of 50 recoil events) [21], and the missing ones were simulated in a similar manner as previous investigations (average of 100 recoil events).

To estimate the amount of overlap, we determined the centre of the cascade at peak damage. The centre was determined as the geometrical centre of the liquid atoms weighted by their kinetic energy. An atom is considered liquid if its kinetic energy and the kinetic energies of its neighbours are above the average kinetic energy at the melting point, in the respective interatomic potential. We calculated the separation distance as the distance between the cascade centre and the centre of the defect cluster. The volume of the cascade (volume of the liquid atoms) was calculated at peak damage, and by assuming a spherical volume (which is a good approximation at low PKA energies), a cascade radius $\left(r_{\mathrm{c}}\right)$ was determined. To analyse the defect number and structure after the cascade, OVITO [30] was used. The Wigner-Seitz analysis implemented in OVITO was used to identify the number of interstitials and vacancies. The number of new defects was analysed as a function of the separation distance, and grouped into bins of $5 \AA$. In each bin, the mean and its standard error was calculated.

To obtain the dislocation structure, the DXA [31] analysis was utilised. Previously, planar voids or open dislocation loops have been investigated in reference [32], and seen to have a similar energy as the vacancy-type dislocation-loops at certain sizes. Therein, planar voids are referred to as "open loops" and dislocation loops as "closed loops". In this article, "loop" refers to a (closed) dislocation loop and "planar void" is used to refer to a loop that has "opened up" in the direction normal to the loop plane. To relate the produced dislocation structures to their stability, formation energies at $0 \mathrm{~K}$ for both types of loops, both types of planar voids, and spherical voids were calculated. The formation energies were calculated following a conjugate gradient minimisation at zero pressure using LAMMPS [33]. Scaling laws corresponding to each cluster configuration, as discussed in reference [32], were fitted to the obtained formation energies. The formation energy of dislocation loops scales with the number of vacancies, $N$, as

$$
E_{\mathrm{f}}=a_{1} \sqrt{N} \ln (N)+a_{2} \sqrt{N}+a_{3},
$$

where the coefficients $a_{i}$ are used as fitting parameters. For planar voids, the formation energy scales as

$$
E_{\mathrm{f}}=a_{1} N+a_{2},
$$

and for voids as

$$
E_{\mathrm{f}}=a_{1} N^{2 / 3} .
$$

\section{Results and discussion}

\subsection{Defect production}

The defect production given as the number of Frenkel pairs $\left(N_{\mathrm{FPs}}\right)$ by $5 \mathrm{keV}$ and $10 \mathrm{keV}$ recoils in pristine material can be found in Table 1, for both temperatures and both interatomic potentials. The cascade volume radius $\left(r_{\mathrm{c}}\right)$ for the same parameters is also found in the same table. For both potentials we can observe that more defects are produced in $10 \mathrm{keV}$ cascades than in $5 \mathrm{keV}$ cascades, and that more defects are formed in the M07B 


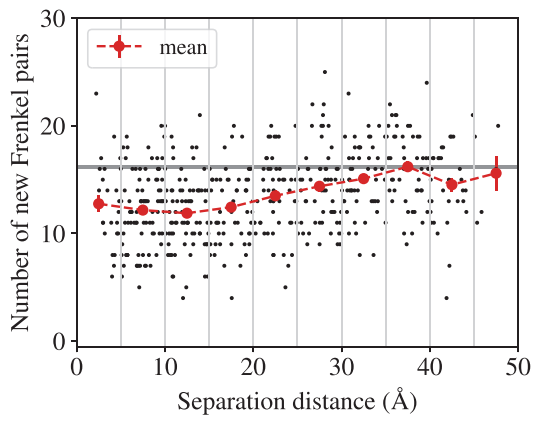

(a) $\langle 100\rangle$ loop

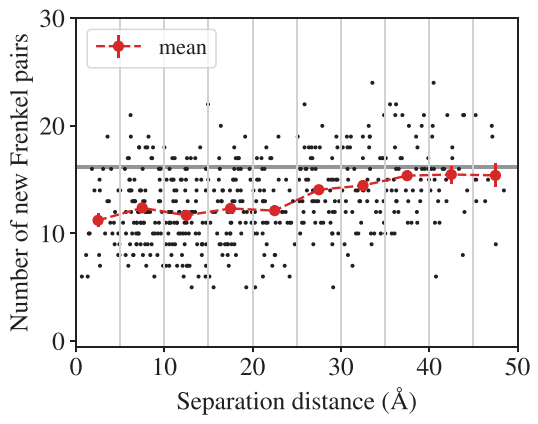

(b) $1 / 2\langle 111\rangle$ loop

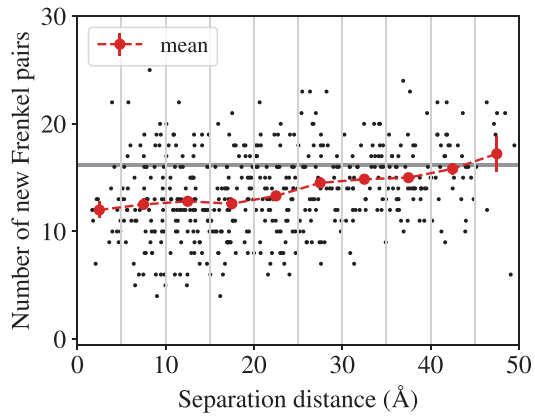

(c) Spherical void

Fig. 1. Defect production for $5 \mathrm{keV}$ cascades in the vicinity of the different $1.8 \mathrm{~nm}$ vacancy-type defects at $0 \mathrm{~K}$ in the $\mathrm{M} 07 \mathrm{~B}$ potential. The individual small black dots are the raw data and the larger red dots are their mean in the certain intervals.

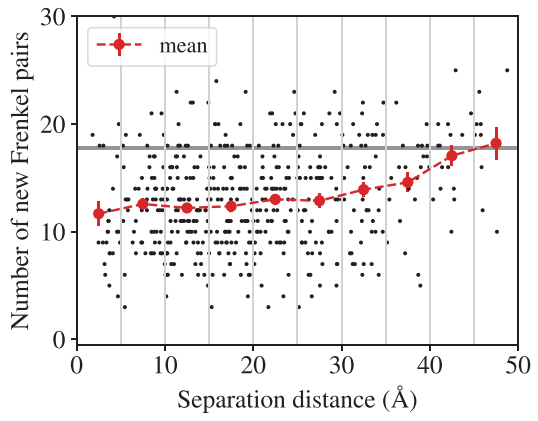

(a) $\langle 100\rangle$ loop

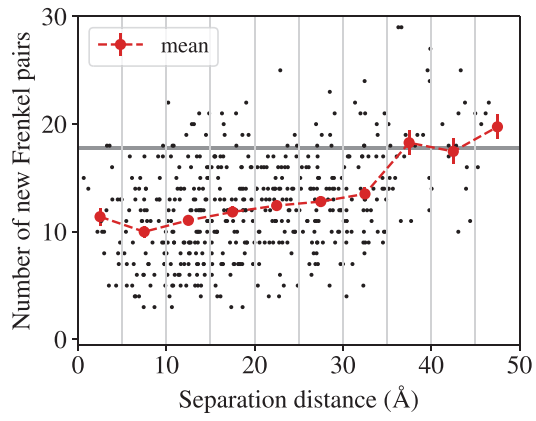

(b) $1 / 2\langle 111\rangle$ loop

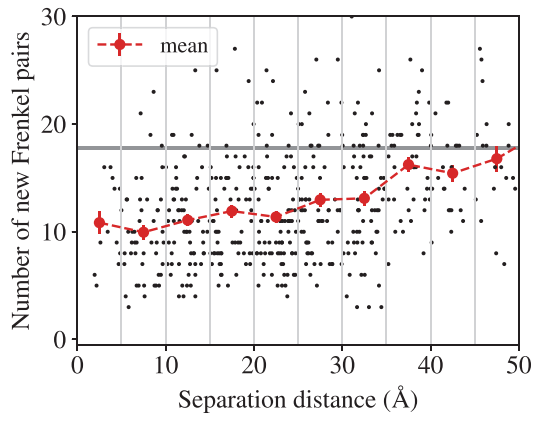

(c) Spherical void

Fig. 2. Defect production for $10 \mathrm{keV}$ cascades in the vicinity of the different $2.5 \mathrm{~nm}$ vacancy-type defects at $300 \mathrm{~K}$ in the A04 potential. The individual small black dots are the raw data and the larger red dots are their mean in the certain intervals.

Table 1. Number of Frenkel pairs $\left(N_{\mathrm{FPs}}\right)$ and cascade radius $\left(r_{\mathrm{c}}\right)$ in pristine material for the investigated interatomic potentials (Pot.), temperatures $(T)$ and recoil energies $(E)$.

\begin{tabular}{lllll}
\hline Pot. & $E(\mathrm{keV})$ & $T(\mathrm{~K})$ & $N_{\mathrm{FPs}}$ & $r_{\mathrm{c}}(\AA)$ \\
\hline A04 & 5 & 0 & $13.1 \pm 0.5[21]$ & $16.3[21]$ \\
& & 300 & $10.8 \pm 0.4[21]$ & $17.6[21]$ \\
& \multirow{2}{*}{10} & 0 & $22.6 \pm 0.5$ & 20.4 \\
M07B & 5 & 300 & $17.8 \pm 0.4$ & 21.4 \\
& \multirow{2}{*}{10} & 0 & $16.2 \pm 0.5[21]$ & $14.6[21]$ \\
& & 0 & $12.8 \pm 0.5[21]$ & $15.7[21]$ \\
& & 300 & $27.0 \pm 0.5$ & 17.8 \\
\end{tabular}

potential than in the A04 potential. At the higher temperature, fewer defects are produced than at $0 \mathrm{~K}$. The cascade radius, on the other hand, is larger in the A04 potential than in the M07B potential (due to differences in the predicted melting points), and larger at the higher temperature. These parameter and interatomic potential specific differences have earlier been discussed in for instance $[1,18,34,35]$.

As it can be assumed, we observed that if the separation distance is large, i.e. the cascade centre is far from the defect cluster, the defect production is not affected by the pre-existing defect. This can be seen in Figure 1, for $5 \mathrm{keV}$ recoils and the size $1.8 \mathrm{~nm}$ at $0 \mathrm{~K}$ in the M07B potential, and in Figure 2, for $10 \mathrm{keV}$ recoils and the size $2.5 \mathrm{~nm}$ at $300 \mathrm{~K}$ in the A04 potential. The same trend can also be seen for the other sizes at both temperatures in both potentials. The defect production in this region is on average the same as in pristine material, as also seen for the interstitial-type defects previously [21]. At smaller separation distances, where overlap happens, there is an effect on the defect production. For the single vacancy, no statistically significant difference was seen for neither of the potentials nor at any temperature or PKA energy. For the larger defect structures, we observe a decrease in defect production at serious overlap for all investigated parameters in both potentials, as seen in Figure 3.

Studying the general trend of the effect of separation distance on the defect production in all investigated setups, the reduction is not as visible as for interstitialtype defects [21]. This smaller effect makes it more difficult to draw any quantitative conclusions. For almost all parameters we observe at full and almost full overlap a quite stable defect production, described in the next paragraph. At larger separation distances, we start to see an increase in defect production, until no overlap effect is seen. Generally, a larger defect size or a higher PKA energy (leading to a greater cascade volume) will shift the limit of no overlap, where the number of new defects is the same as in the pristine material, towards higher separation distances. The separation distance after which there 


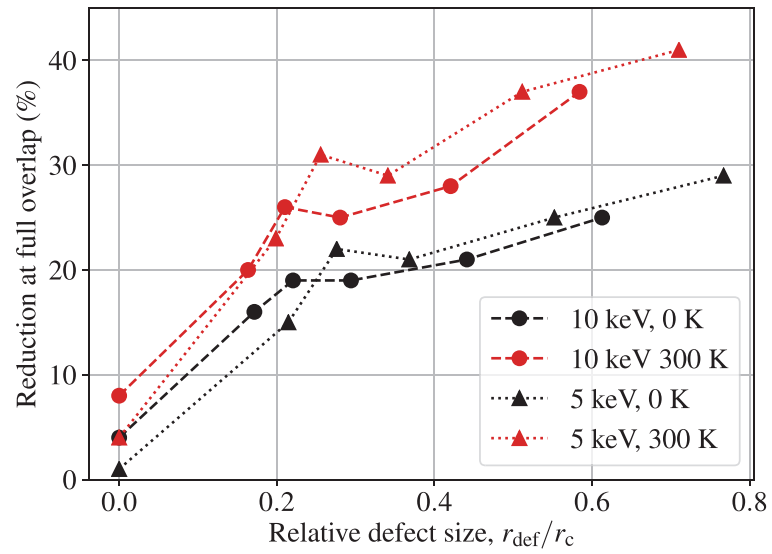

(a) A04 potential

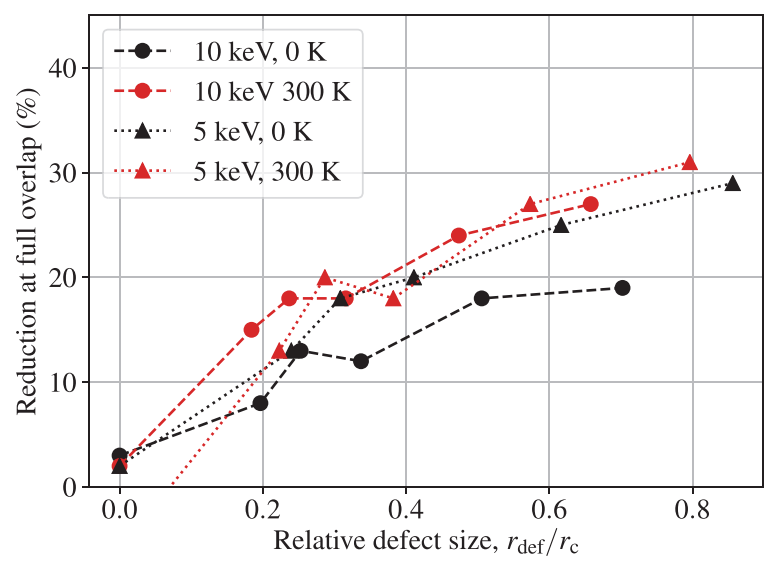

(b) M07B potential

Fig. 3. Defect production reduction percentage at serious cascade overlap in both the A04 potential (a) and the M07B potential (b). The $x$-axis is scaled by the cascade radius to account for the dependence on energy. The values for the point defect and for the two smallest sizes are only for the spherical void configuration, the others are the averages over all three different defect types.

is no effect can be seen to be between $2 \mathrm{~nm}$ and $4 \mathrm{~nm}$, corresponding very well to the sum of the defect radius and the cascade radius (between $2 \mathrm{~nm}$ and $3.5 \mathrm{~nm}$ ).

Looking at the defect production where significant overlap is happening in all cases, here defined as the defect production up to a separation distance of $1.5 \mathrm{~nm}$, some general observations can be made. We see that the defect production in both potentials is similar for all initial defects structures, when the cluster radius is the same. This indicates that the defect production is independent of defect structure when it is completely enclosed by the cascade volume. The average reduction for all the investigated sizes can be seen in Figure 3. The values for the single vacancy and for the two smallest sizes are only for the spherical void configuration, i.e. the statistics are about one third compared to the rest. For the three larger sizes, the average of all three defect types is shown. For both interatomic potentials we can observe a larger reduction in the number of produced defects when the size of the defect cluster is increased. In the A04 potential, an increase in temperature will increase the reduction, whereas in the M07B potential the same trend is seen for the higher recoil energy but not as clearly for the lower energy. On average we also observe a higher reduction in the A04 potential compared to the M07B potential. However, the statistical fluctuations are large, as is visible in Figures 1 and 2.

\subsection{Defect transformation}

To investigate the transformation of defects under irradiation, we can look at two factors, the cluster size evolution and the defect structure transformation. In the first part, the largest cluster after the recoil event is analysed in form of number of vacancies in this cluster. The cutoff for considering two vacancies to belong to the same cluster was the distance in the middle of the second and third nearest neighbours, which is commonly used for vacancies. In the second part, the dislocations identified by the DXA after the recoil event are studied.

The size evolution follows a similar trend as the defect production. When the recoil is far from the defect cluster, almost no effect is seen. There is on average a small reduction, as some interstitials may recombine with the vacancy-type defect clusters, which can be explained by the interstitials being pushed out from the cascade centre [36]. At all distances, on average, we observe a reduction in size for all investigated defects clusters, except for the single vacancy. In some individual cases an increase in size can be observed, where the vacancyrich cascade centre [36] is combined with the vacancy rich defects. For both potentials we observe a greater reduction in size for more energetic recoils, however, the reduction is much greater in the A04 potential compared to the M07B potential. The reduction is roughly 10 and 20 defects for the A04 potential for both energies, and the corresponding reduction in the M07B potential is about 5 and 10 defects. From the results we also observed that the largest reduction is not at direct overlap, especially not for the spherical voids. The overall reduction in cluster size can be explained by two factors. At full overlap, the molten cascade centre can destroy the underlying features, and spread out the vacancies. At larger distances, the interstitials pushed away from the cascade core can recombine with some of the vacancies found in the vacancy-type defect. Both of these will effectively reduce the size of the largest cluster (the initial defect structure).

To understand the dislocation structure transformation of the vacancy-type dislocation loops and the production of loops in the spherical void simulations, their relative stability is useful. The stability curves in terms of formation energies of the dislocation loops, planar voids and spherical voids of different sizes are shown in Figure 4. In both potentials, the spherical void is more stable than both loops of different Burgers vectors and both planar voids. The planar $1 / 2\langle 111\rangle$ void has in both potentials the same or higher formation energy than the dislocation loop at all sizes. This indicates that the small loops could open up, but the larger loops should not open up. For 


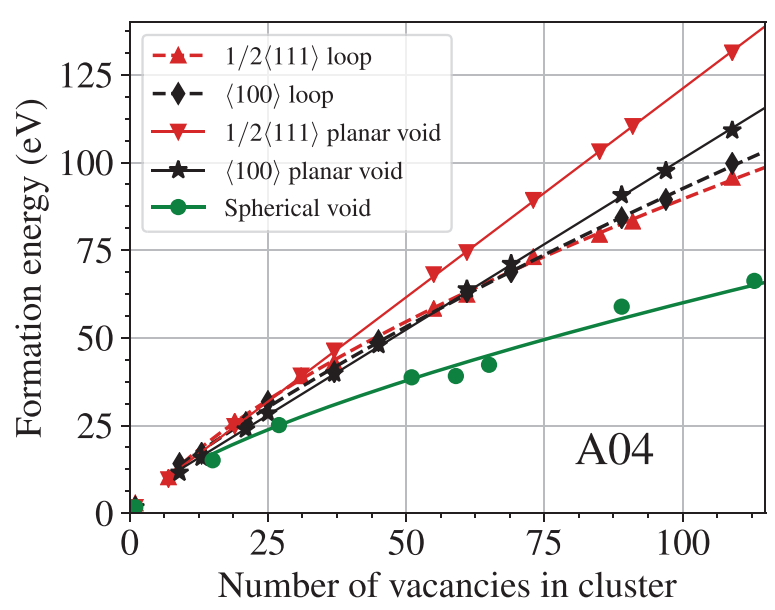

(a) A04 potential

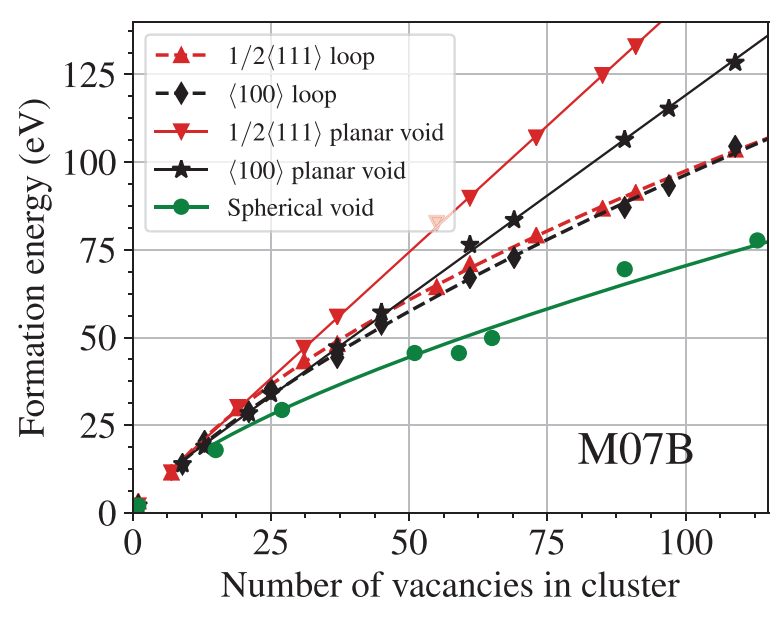

(b) M07B potential

Fig. 4. Formation energy of the loops of both Burgers vectors, planar voids of both orientations as well as the formation energy for spherical voids of different sizes. The results in the A04 potential is found in subfigure (a) and M07B potential in subfigure (b).

the $\langle 100\rangle$ loop, on the other hand, the planar void is at small sizes more stable than the dislocation loop in the A04 potential, and has a similar or higher energy than the dislocation loop in the M07B potential. This indicates that the smallest loop, especially in the A04 potential, can open up during irradiation. An important factor to account for, is that even though the planar void has a higher energy than the dislocation loop, a hybrid between a planar void and a spherical void can still and will have a lower energy than the dislocation loops.

To study the transformation of the defect clusters in form of dislocation structure, we focus on two parts. The main focus is on the transformation of the structures at full overlap (up to a separation distance of $1.5 \mathrm{~nm}$ ) the effect of separation distance on the transformation is also briefly discussed. In the following, a mixed loop means either a combination of both types of dislocation loops or one partial loop connected with an unidentified defect according to DXA. The probabilities for different outcomes for both types of loops for all parameters can be found in the supplementary material online. Here, only the main findings are described and the reader is referred to the supplementary material for detailed results.

For the smallest dislocation loops at both temperatures, both energies and both interatomic potentials, at full overlap there is almost never any dislocations remaining according to the DXA. As described earlier, the cluster size reduction is between 5 and 20 defects, which leads to a very small remaining cluster, which cannot be recognised or even interpreted anymore as a dislocation loop. Looking at the exact positions of the vacancies, we can also observe that for the smallest loops, the vacancies have either been completely scattered, or reformed into a more complex three-dimensional structure.

For the intermediate-sized dislocation loops we can observe, according to the DXA, that there is a very small probability for a loop to survive in the A04 potential at both temperatures. In the M07B potential, on the other hand, the dislocation loops are more likely to survive the recoil event at both temperatures. For the initially $\langle 100\rangle$ loop we observe some $\langle 100\rangle$ loops and some mixed loops in the M07B potential. For the initially $1 / 2\langle 111\rangle$ loops, both types of loops as well as mixed loops are formed in the M07B potential. Interestingly, there are more or equal amounts of $\langle 100\rangle$ loops as mixed loops, and $1 / 2\langle 111\rangle$ loops are the least likely to form. At this size, in the M07B potential, we can clearly observe dislocation loop transformation. Looking at the vacancy positions, a large cluster of vacancies is gathered in the centre of the preexisting defect, quite densely, more so at $5 \mathrm{keV}$ than at $10 \mathrm{keV}$, and more in the M07B potential than in the A04 potential, according to the cluster size reduction. In the A04 potential, we can see complex three-dimensional structures, whereas in the M07B the structures are more planar, which explains why dislocations are more readily found in the M07B potential.

For the largest dislocations, we observe in the majority of the cases some dislocation structure after the recoil event in the M07B potential for all parameters and in the A04 potential at $300 \mathrm{~K}$. In the A04 potential at $0 \mathrm{~K}$ we do not observe almost any dislocation structures. At $300 \mathrm{~K}$ in the A04 potential mainly mixed loops and some $\langle 100\rangle$ loops are seen for both initial dislocation types, and a few $1 / 2\langle 111\rangle$ loops are seen after the cascade fully overlaps with the $1 / 2\langle 111\rangle$ loop. In the M07B potential we observe mainly mixed loops and some $\langle 100\rangle$ loops at $0 \mathrm{~K}$, and in the $1 / 2\langle 111\rangle$ case some $1 / 2\langle 111\rangle$ loops. At $300 \mathrm{~K}$, mainly $\langle 100\rangle$ loops are seen, also for the initially $1 / 2\langle 111\rangle$ loop. For both initial Burgers vectors we also see mixed loops and in the $1 / 2\langle 111\rangle$ case some $1 / 2\langle 111\rangle$ loops. Again, we observe a transformation of the $1 / 2\langle 111\rangle$ loops to $\langle 100\rangle$ loops in both potentials, even though both loops have very similar energies. Looking at the vacancy positions, we can see that at $0 \mathrm{~K}$ in the A04 potential, where almost no dislocations are formed, there are mainly vacancy-rich three-dimensional defect structures. As seen for the intermediate loops, the defect clusters after the cascade are more planar in the M07B potential at $0 \mathrm{~K}$ 
also for the largest loops, explaining the large fraction of dislocations and mixed dislocations seen.

Looking at the effect of separation distance on the dislocation evolution, the following general trend can be observed for both loops in both potentials at all investigated parameters, except the few cases described later. As it can be assumed, the probability of remaining in the original structure increases as the separation distance increases. The probability of destruction or change in Burgers vector will decrease as the distance is growing. An exception to this was the smallest $\langle 100\rangle$ loop at $300 \mathrm{~K}$, where no dislocations were left after the recoil event. The explanation can be seen from the formation energy graph, Figure 4 , as the $\langle 100\rangle$ planar void is equally or more stable than the $\langle 100\rangle$ dislocation loop. Only the latter can be identified by the DXA. Looking at the vacancy positions, we could verify that the loops were untouched by the cascade, and the only change was the switch from a dislocation structure to a planar void structure. A similar phenomenon was seen for some of the intermediate loops in the A04 potential, where there was a large probability for no loop after the recoil, even at the furthest distance. At this size, the stability of the dislocation loops and the planar voids are very close to each other, however, this is not seen in the M07B potential, as the dislocation loop is much more stable than the planar void. This indicates that the thermal motion and/or the shockwave of the cascade can trigger a transformation towards a void-like structure.

Investigating the dislocation production when the cascade overlaps with voids, we can see that there is a chance to create perfect $\langle 100\rangle$ loops in both potentials. There is also a probability for mixed loops, in this case mainly a dislocation segment bound to the void. These probabilities are found in the supplementary material. Most of the differently sized voids have their highest probability of creating dislocations at full overlap, however, the larger ones have a higher probability at around $2 \mathrm{~nm}$ separation distance. This separation distance corresponds to the cascade centre being very close to the edge of the void. Many of the produced dislocations close to large voids are of mixed type, so mainly dislocations that are bound to the voids, however, the dislocation size can be substantial.

\section{Conclusions}

In this paper, we studied the effect of vacancy-type defects on the defect production and defect transformation, when an energetic cascade is in their vicinity. We observed that the defect production was affected by a nearby vacancyrich defect cluster. The defect production was reduced by a few tens of percent when the cascade was fully or partially overlapping with the pre-existing defect cluster. The reduction was, however, not as drastic as previously seen for similar sized interstitial-type defect clusters [21]. The defect production reduction was not dependent on the defect structure, only on the defect cluster radius, when the cascade volume fully enclosed the defect. The effect of the nearby cluster was seen up to a separation distance corresponding to the defect radius plus the cascade radius. We observed for all cluster sizes, except for the single vacancy, that the largest cluster was reduced in size after a fully overlapping cascade. The reduction was dependent on PKA energy and interatomic potential, but not on initial size. We observed that some dislocation loops did open up to a planar void structure at room temperature, due to their relative stability. Additionally, we could see that the $\langle 100\rangle$ loops did not transform into $1 / 2\langle 111\rangle$ loops, however, the $1 / 2\langle 111\rangle$ loops were seen to quite often transform into $\langle 100\rangle$ loops when the cascade overlapped fully with the dislocation. Cascades in the vicinity of voids were also seen to produce dislocations, mainly dislocation segments connected with the void. The dislocation produced could be substantial in size. We also observed for some void sizes a quite high production probability of perfect $\langle 100\rangle$ loops. Our results, both the defect production bias in the vicinity of vacancy-rich defects and the transformation probabilities can, in addition to previous results on interstitial-rich defects, be transferred to higher-scale simulation methods.

Open access funding provided by University of Helsinki including Helsinki University Central Hospital. This work has been carried out within the framework of the EUROfusion Consortium and has received funding from the Euratom research and training programme 2014-2018 and 2019-2020 under grant agreement No 633053. The views and opinions expressed herein do not necessarily reflect those of the European Commission. Computer time granted by the IT Center for Science - CSC Finland and the Finnish Grid and Cloud Infrastructure (persistent identifier urn:nbn:fi:research-infras-2016072533) are gratefully acknowledged.

\section{Supplementary material}

Supplementary material containing the created dislocations at full overlap for all investigated parameters can be found online at https://doi.org/10.1140/epjb/ e2019-100240-3.

\section{Author contribution statement}

F.G. carried out the recoil simulations, analysed the data and wrote the initial draft of the paper. J.B. created the input files, ran the stability simulations and carried out the data analysis. K.N. initiated the project. All authors contributed to interpret the data, draw the conclusions and write the article.

Open Access This is an open access article distributed under the terms of the Creative Commons Attribution License (http://creativecommons.org/licenses/by/4.0), which permits unrestricted use, distribution, and reproduction in any medium, provided the original work is properly cited.

\section{References}

1. L. Malerba, J. Nucl. Mater. 351, 28 (2006) 
2. K. Nordlund, S.J. Zinkle, A.E. Sand, F. Granberg, R.S. Averback, R.E. Stoller, T. Suzudo, L. Malerba, F. Banhart, W.J. Weber et al., J. Nucl. Mater. 512, 479 (2018)

3. M.L. Jenkins, Z. Yao, M. Hernández-Mayoral, M.A. Kirk, J. Nucl. Mater. 389, 202 (2009)

4. Z. Yao, M.L. Jenkins, M. Hernández-Mayoral, M.A. Kirk, Philos. Mag. 90, 4634 (2010)

5. R.S. Averback, R. Benedek, K.L. Merkle, Phys. Rev. B 18, 4171 (1978)

6. B.C. Masters, Philos. Mag. 11, 881 (1965)

7. M.L. Jenkins, C.A. English, B.L. Eyre, Philos. Mag. A 38, $97(1978)$

8. M.C. Marinica, F. Willaime, J.P. Crocombette, Phys. Rev. Lett. 108, 025501 (2012)

9. Y.N. Osetsky, D.J. Bacon, V. Mohles, Philos. Mag. 83, 3623 (2003)

10. S.M. Hafez Haghighat, J. Fikar, R. Schäublin, J. Nucl. Mater. 382, 153 (2008)

11. D. Terentyev, D.J. Bacon, Y.N. Osetsky, J. Phys.: Condens. Matter 20, 445007 (2008)

12. G. Bonny, D. Terentyev, L. Malerba, J. Nucl. Mater. 416, 74 (2011)

13. R. Schäublin, S.M. Hafez Haghighat, J. Nucl. Mater. 442, S648 (2013)

14. F. Granberg, D. Terentyev, K.O.E. Henriksson, F. Djurabekova, K. Nordlund, Fusion Sci. Technol. 66, 288 (2014)

15. F. Granberg, D. Terentyev, K. Nordlund, J. Nucl. Mater. 460, 29 (2015)

16. K. Vörtler, N. Juslin, G. Bonny, L. Malerba, K. Nordlund, J. Phys.: Condens. Matter 23, 355007 (2011)

17. F. Granberg, K. Nordlund, M.W. Ullah, K. Jin, C. Lu, H. Bei, L.M. Wang, F. Djurabekova, W.J. Weber, Y. Zhang, Phys. Rev. Lett. 116, 135504 (2016)

18. J. Byggmästar, F. Granberg, K. Nordlund, J. Nucl. Mater. 508, 539 (2018)
19. F. Granberg, J. Byggmästar, A.E. Sand, K. Nordlund, Europhys. Lett. 119, 56003 (2017)

20. A.E. Sand, J. Byggmästar, A. Zitting, K. Nordlund, J. Nucl. Mater. 511, 74 (2018)

21. J. Byggmästar, F. Granberg, A.E. Sand, A. Pirttikoski, R. Alexander, M.C. Marinica, K. Nordlund, J. Phys.: Condens. Matter 31, 245402 (2019)

22. X. Wang, N. Gao, Y. Wang, H. Liu, G. Shu, C. Li, B. Xu, W. Liu, J. Nucl. Mater. 519, 331 (2019)

23. K. Nordlund, M. Ghaly, R.S. Averback, M. Caturla, T. Diaz de la Rubia, J. Tarus, Phys. Rev. B 57, 7570 (1998)

24. M. Ghaly, K. Nordlund, R.S. Averback, Philos. Mag. A 79, 795 (1999)

25. K. Nordlund, Comput. Mater. Sci. 3, 456 (1995)

26. G.J. Ackland, M.I. Mendelev, D.J. Srolovitz, S. Han, A.V. Barashev, J. Phys.: Condens. Matter 16, S2629 (2004)

27. L. Malerba, M. Marinica, N. Anento, C. Björkas, H. Nguyen, C. Domain, F. Djurabekova, P. Olsson, K. Nordlund, A. Serra et al., J. Nucl. Mater. 406, 38 (2010)

28. C. Björkas, K. Nordlund, Nucl. Instrum. Methods Phys. Res. Sec. B 267, 1836 (2009)

29. H.J.C. Berendsen, J.P.M. Postma, W.F. van Gunsteren, A. DiNola, J.R. Haak, J. Chem. Phys. 81, 3684 (1984)

30. A. Stukowski, Modell. Simul. Mater. Sci. Eng. 18, 015012 (2010)

31. A. Stukowski, V.V. Bulatov, A. Arsenlis, Modell. Simul. Mater. Sci. Eng. 20, 085007 (2012)

32. M.R. Gilbert, S.L. Dudarev, P.M. Derlet, D.G. Pettifor, J. Phys.: Condens. Matter 20, 345214 (2008)

33. S. Plimpton, J. Comput. Phys. 117, 19 (1995)

34. R.E. Stoller, J. Nucl. Mater. 276, 32 (2000)

35. D. Terentyev, C. Lagerstedt, P. Olsson, K. Nordlund, J. Wallenius, C. Becquart, L. Malerba, J. Nucl. Mater. 351, 77 (2006)

36. B.N. Singh, A.J.E. Foreman, Philos. Mag. A 66, 990 (1992) 